\title{
Cultural and linguistic adaptation of the multi-dimensional OXCAP-MH for outcome measurement of mental health among people living with HIV/AIDS in Uganda: the Luganda version
}

Kenneth R. Katumba ${ }^{1 *}$ (D) Yoko V. Laurence ${ }^{2}$, Patrick Tenywa ${ }^{3}$, Joshua Ssebunnya ${ }^{4}$, Agata Laszewska ${ }^{5}$, Judit Simon ${ }^{5,6}$, Anna Vassall2, Eugene Kinyanda ${ }^{3}$ and Giulia Greco ${ }^{2}$

\begin{abstract}
Background: It is rare to find HIV/AIDS care providers in sub-Saharan Africa routinely providing mental health services, yet 8-30\% of the people living with HIV have depression. In an ongoing trial to assess integration of collaborative care of depression into routine HIV services in Uganda, we will assess quality of life using the standard EQ-5D-5L, and the capability-based OxCAP-MH which has never been adapted nor used in a low-income setting. We present the results of the translation and validation process for cultural and linguistic appropriateness of the OxCAP-MH tool for people living with HIV/AIDS and depression in Uganda.

Methods: The translation process used the Concept Elaboration document, the source English version of OxCAP$\mathrm{MH}$, and the Back-Translation Review template as provided during the user registration process of the OxCAP-MH, and adhered to the Translation and Linguistic Validation process of the OxCAP-MH, which was developed following the international principles of good practice for translation as per the International Society for Pharmacoeconomics and Outcomes Research's standards.

Results: The final official Luganda version of the OxCAP-MH was obtained following a systematic iterative process, and is equivalent to the English version in content, but key concepts were translated to ensure cultural acceptability, feasibility and comprehension by Luganda-speaking people.
\end{abstract}

Conclusion: The newly developed Luganda version of the OxCAP-MH can be used both as an alternative or as an addition to health-related quality of life patient-reported outcome measures in research about people living with HIV with comorbid depression, as well as more broadly for mental health research.

Keywords: Capability approach, Mental health, Quality of life, HIV/AIDS, Uganda, PROMs

\footnotetext{
* Correspondence: Kenneth.Katumba@mrcuganda.org

${ }^{1}$ MRC/UVRI \& LSHTM Uganda Research Unit (Social Aspects of Health), Plot

51-59 Nakiwogo Road, Entebbe +256, Uganda

Full list of author information is available at the end of the article
}

\section{Springer Open}

(c) The Author(s). 2021 Open Access This article is licensed under a Creative Commons Attribution 4.0 International License, which permits use, sharing, adaptation, distribution and reproduction in any medium or format, as long as you give appropriate credit to the original author(s) and the source, provide a link to the Creative Commons licence, and indicate if changes were made. The images or other third party material in this article are included in the article's Creative Commons licence, unless indicated otherwise in a credit line to the material. If material is not included in the article's Creative Commons licence and your intended use is not permitted by statutory regulation or exceeds the permitted use, you will need to obtain permission directly from the copyright holder. To view a copy of this licence, visit http://creativecommons.org/licenses/by/4.0/. 


\section{Introduction}

Studies in sub-Saharan Africa report depression rates of 8-30\% [1-4] among the 25 million people living with HIV [5]. However, the majority of HIV/AIDS care providers in the region do not routinely provide mental health services [1]. In Uganda the call for the integration of mental health and other chronic conditions in HIV care by the National HIV and AIDS strategic plan 2015-2020 [6] has received support in the form of the Ministry of Health $(\mathrm{MoH})$ consolidated guidelines for the prevention and treatment of HIV in Uganda [7]. These guidelines have for the first time called for the assessment and management of depression in people living with HIV [6, 7].

Depression is associated with lower quality of life [8] and a number of negative clinical and behavioural outcomes, including rapid HIV disease progression leading to mortality $[9,10]$, poor adherence to HIV treatment $[3,11-13]$, risky sexual behaviour $[11,14-16]$ and increased utilisation of health facilities [3,11, 13-15].

The HIV + D study is an on-going cluster randomized controlled trial led by the MRC/UVRI \& LSHTM Uganda Research Unit in partnership with the STD/ AIDS control programme of the Ugandan ministry of health, that evaluates the effectiveness of integrating stepped, collaborative care of depression into routine HIV services in Uganda. An economic evaluation is planned alongside the trial and it was thus necessary to identify an appropriate outcome measure to use in the cost-effectiveness analysis.

Patient-reported outcome measures (PROMs) have been used to evaluate healthcare interventions via costeffectiveness analyses $[13,17,18]$. Health-related quality of life (HRQoL) PROMs are a systematic approach for obtaining meaningful, subjective accounts from patients on their quality of life [19] and satisfaction with health services [13, $17,18]$. They can be generic and therefore used across diseases and conditions, such as the 36- and 12-item Short Form Surveys (SF-36, SF-12) [20, 21], and the EQ-5D [22]. The sensitivity and appropriateness of these HRQoL PROMs in more specific populations such as those with comorbidities has however been questioned [13]. The selection of an appropriate HRQoL PROM is therefore dependent on the target population, the nature of the intervention, and its ultimate use, with many trials supplanting a disease-specific HRQoL PROM that will be more sensitive to treatment with a generic HRQoL PROM that is more comparable across populations and disease-conditions [13, 17].

Generic HRQoL PROMs have been used globally [23, 24], while disease-specific HRQoL PROMS have mainly been developed and used in Europe and other highincome countries to assess quality of life in patients with depression [25-28]. No disease-specific HRQoL PROM has however been developed or adapted to assess quality of life in patients with depression in Africa.
Over the years, there has been growing interest in the development and use of PROMs that include a multidimensional understanding of wellbeing. Some of the methodological progress in this area has been grounded in Amartya Sen's capability approach, which focuses on the freedom that people have to be or to do what that they have reason to value, given their personal characteristics and external circumstances [29, 30].. PROMs that are rooted in the capabilities approach include domains that are relevant and important to the quality of life of people with mental health disorders, such as the quality of their relationships, sense of belonging and acceptance, self-perception, autonomy, freedom of choice, and feeling of hope [31]. In the HIV + D trial [32] in Uganda we proposed the use of the Oxford CAPabilities Questionnaire for Mental Health (OxCAP-MH) alongside the EQ-5D-5L to assess both the broader-dimensional and the generic HRQoL of people living with HIV/AIDS and depression.

The OxCAP-MH is a multidimensional outcome measure for mental health interventions, based on the capabilities approach. It was originally developed in the UK and has been translated and adapted so far for the German, Hungarian and Chinese populations [27]. To date the questionnaire has been psychometrically validated in the UK, Germany and Austria for use among populations with mental health disorders [25, 33, 34]. It has also been used in several trial-based mental health economic evaluations [28, 35].

It comprises 16 items which reflect the 10 central capabilities by Nussbaum [30]: social networks, love and support, suitable accommodation, neighbourhood safety, daily activities, losing sleep, enjoy recreation, potential for assault, discrimination, influence local decisions, freedom of expression, appreciate nature, respect and appreciation, planning one's life, imagination and creativity, and access to interesting activities [25, 27]. OxCAP-MH offers a multi-faceted and broad scope for assessing the quality of life of people living with HIV/ AIDs and depression, as it includes aspects of social integration, discrimination and stigma $[25,27]$.

Though the OxCAP-MH has only been validated in high-income settings in Europe, it was chosen for the HIV + D study in Uganda to complement the EQ-5D-5L outcome measure as it captures the effects of the trial beyond health.

In this study we present the results of the translation and the validation process for cultural and linguistic appropriateness of the OxCAP-MH tool for use with people living with HIV/AIDS and depression in Uganda.

\section{Methods}

The translation and the cultural validation process was coordinated by the HIV + D Health Economics team, 
and supported by a team of mental health experts consisting of clinical psychiatrists, clinicians, research assistants and two expert clients. The quality of this process was ensured by adhering to the Translation and Linguistic Validation (TLV) process of the OxCAP-MH as stipulated by the custodian of the tool, which was developed following the international principles of good practice for PROMs' translation as per the International Society for Pharmacoeconomics and Outcomes Research's (ISPOR) standards [36]. We also used the Concept Elaboration document, the source English version of OxCAP-MH, and the Back-Translation Review template as provided during the user registration process of the OxCAP-MH [37].

Figure 1 below outlines the process of translating and validating the OxCAP-MH into Luganda.

Forward translation and forward translation reconciliation The first forward translation from English to Luganda was based on the source English version of the OxCAP$\mathrm{MH}$, and two additional questions derived from the earlier developmental version of the English OxCAP-MH from the paper by Simon et al. (2013) [38]. Guided by the concept elaboration document, the forward translations were carried out by two independent translators: a Health Economist and a Clinical Psychologist. Both translators were native Luganda speakers and proficient in English, with extensive experience in medical translations. From these translations, a reconciled Luganda version (OxCAP-MH Luganda version 1) was produced that most accurately represented the concepts within the source English version.

\section{Back-translation and review}

OxCAP-MH Luganda version 1 was sent for blinded back-translation to three independent Luganda speakers with proficiency in English and experience in translations: a social scientist; a research assistant; and a BSc Information Technology student.

A multi-disciplinary team formed by the translators and three additional anthropologist/social scientists was appointed to review specific queries on wording and produce a reconciled version, and supported by the developers.

\section{Validation workshop}

According to the TLV process, the OxCAP-MH Luganda version 1 was ready to be piloted at this stage, but we added a further step in the process to improve the validity of the translation before piloting. We organized a validation workshop for the wider HIV + D trial team, which brought together clinical psychiatrists, health economists, research assistants, expert clients - patients with both HIV/AIDS and depression but acting as peer leaders to the other patients, and District Health Officers (DHOs). The objectives of the workshop were to discuss and validate by team agreement both the forward and backward translations, terminologies and wording; and to address any other considerations specific to people living with HIV/AIDS and depression. Consensus was reached on a second version (OxCAP-MH Luganda version 2). None of the key concepts of the OxCAP-MH was changed during this workshop, but concerns about the local applicability of concepts like 'enjoying nature', 'interesting forms of activity', and 'freedom to use imagination to express oneself were cited during this workshop.

\section{Pilot testing (cognitive debriefing) and review}

The aim of the pilot testing was to confirm whether the OxCAP-MH Luganda version 2 was accurately understood and acceptable to a sampled population of people living with HIV and depression. We randomly selected 20 participants of the main HIV + D trial pilot from $4 \mathrm{fa}-$ cilities. The participants were contacted by the health facility supervisors in the HIV $+\mathrm{D}$ trial by telephone, or via community contacts.

Two strategies were compared for delivering and validating the tool: interviewer-administered (IA), where the interviewer administered the tool to the respondents one at a time, sequentially, capturing the responses on the paper questionnaires; and self-administered (SA) [38], where two interviewers guided a group of respondents through each question, and the respondents wrote their own responses on the paper questionnaires independently. For respondents that could not write, the second interviewer documented their responses. IA and SA questionnaires were identical.

The pilot was carried out after obtaining appropriate institutional and statutory ethical approvals. All study participants gave written informed consent, and those that couldn't sign used their fingerprint in the presence of a witness.

Findings from the pilot were reviewed by the trial team and by the custodian of the OxCAP-MH. In addition to amendments to the Luganda version, the official English version of the OxCAP-MH was also slightly modified (OxCAP-MH English (UK) 2020) to accommodate the broader international context, e.g. for Luganda-speaking populations.

\section{Forward and confirmatory back-translation of version 2}

Following the pilot, key questions with complex meanings were reworded to fit the local context and to be understood by study participants without altering the original meaning of the questions. This was done by agreement between the HIV + D Health Economics team and the tool's custodian. 


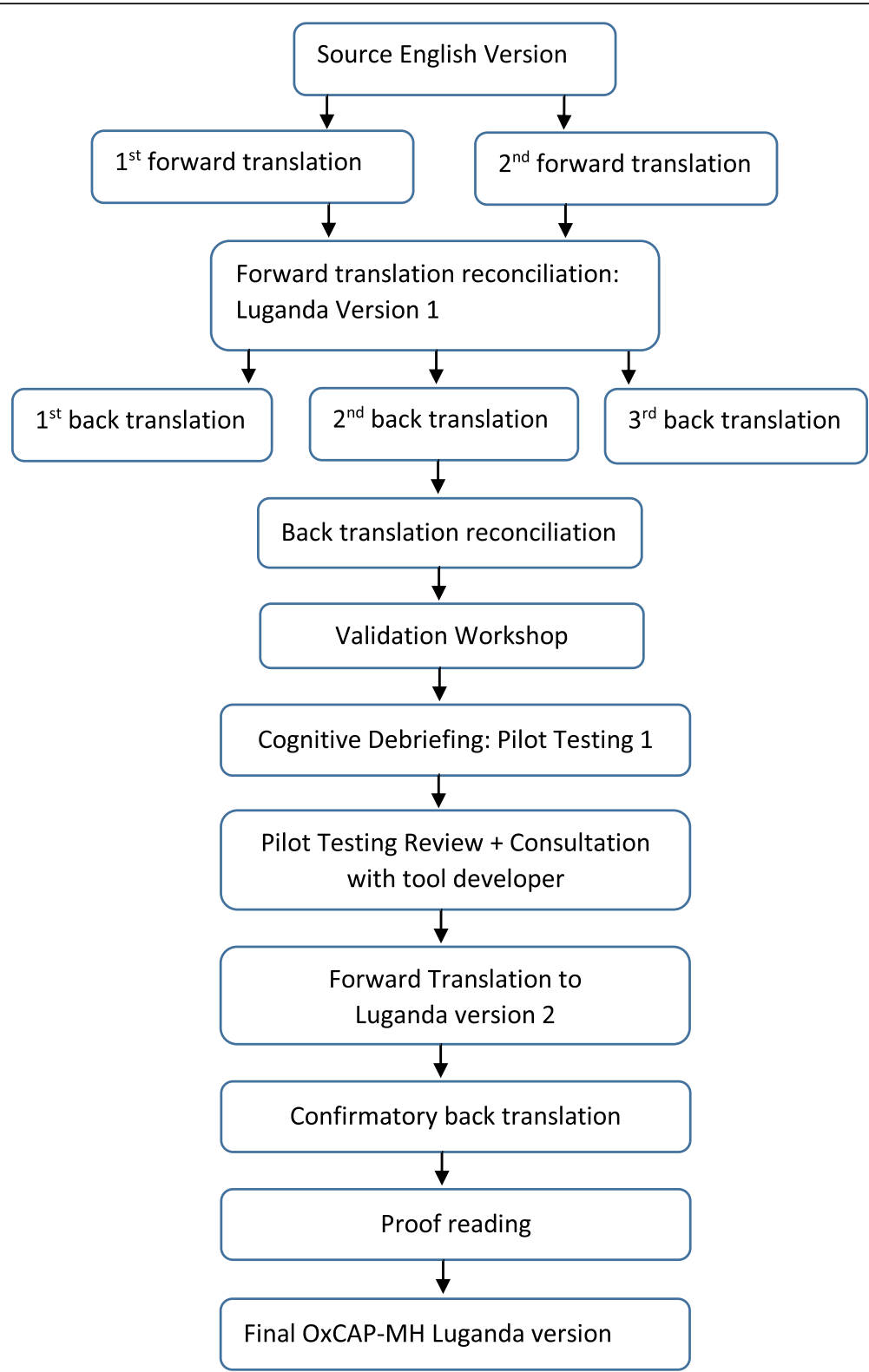

Fig. 1 Translation of the Source OxCAP-MH English version into Luganda according to the Translation and Linguistic Validation process

These questions were back translated from Luganda into English by two independent and blinded social science researchers, and the reconciled OxCAP-MH Luganda version 2 was assessed for consistence and flow by the HIV + D Health Economics team.

\section{Proofreading and review}

Using the Concept Elaboration guidance document, the OxCAP-MH Luganda version 2 was proof-read by a co-investigator of the $\mathrm{HIV}+\mathrm{D}$ trial, who was not involved in any other part of the OxCAP-MH validation process. Review of the feedback created the final OxCAP-MH Luganda version 3 which following a final round of consultation with the tool's custodian, was approved as the official Luganda version of the OxCAP-MH and is now accessible for further use at https://healtheconomics.meduniwien.ac.at/downloads/ oxcap-mh/.

\section{Results}

The official Luganda version of the OxCAP-MH presented in this paper is equivalent to the latest source English version and contains 16 items. Two questions (on home ownership and life expectancy) were not included. The question on life expectancy was kept in the trial patient questionnaire separately from the OxCAP- 
$\mathrm{MH}$, as this was deemed potentially informative in the context of the main HIV $+\mathrm{D}$ trial analysis.

Twenty-nine phrases were translated from the source English questionnaire into Luganda. Of these, 16 were questions, two were additional questions not included in the scoring, and four were instruction phrases. Six were different response options, and one was a sentence to explain the aim of the questionnaire at the beginning.

In following the formal process as shown by Fig. 1, we produced three versions of the Luganda OxCAP-MH, the third version being the final officially accepted version. The first version was created after reconciliation of the two independent forward translations; the second version after the back translation, validation workshop, cognitive debriefing, a second forward translation and confirmatory back translations, and changing seven out of the 29 phrases (24\%); and the final version after proofreading.

Three men and 15 women from four health centres participated in the pilot. Even after replacement and follow-up phone calls, we were not able to successfully reach a fifth respondent in two of the health facilities. The mean age of the participants was 43 years (range 23-58). The characteristics of the participants are detailed in Table 1. Each interview session lasted an average of 5-13 min per participant for the interviewer- administered questionnaires and $30 \mathrm{~min}$ for the selfadministered questionnaires.

Twelve of the sixteen questions (75\%) were easy for the participants to understand. As shown in Table 2 below, the participants found it difficult to understand and answer the initially translated questions on "appreciating nature", "imagination and creativity", and "access". The Translation Coordination Team also expressed concern about potential confusion around the same three concepts, as well as the questions about "daily activities" and "enjoying" recreation. Solutions to these concerns were found after iterative discussions with the custodian of the OxCAP-MH.

At the end of the qualitative analysis of the pilot study results, 11 changes were proposed to the first Luganda version - nine to questions, one to a response option, and one to a particular word. In total, seven of the suggested changes (64\%) were agreed and incorporated in the second Luganda version, resulting in a total of six changed phrases and one changed word.

Some of the proposed changes referred to concepts that were culturally inappropriate or non-existent in $\mathrm{Lu}$ ganda, thereby making it hard to get equivalent translations. These concepts included appreciating nature; imagination and creativity; enjoying recreation; and access.

Table 1 Characteristics of the pilot interview participants

\begin{tabular}{|c|c|c|c|c|c|c|c|}
\hline \multirow[t]{2}{*}{ Health Facility } & \multirow[t]{2}{*}{ Participant Id } & \multirow[t]{2}{*}{ Age } & \multirow[t]{2}{*}{ Sex } & \multirow[t]{2}{*}{ Interview Strategy } & \multirow[t]{2}{*}{ Interview Time } & \multicolumn{2}{|c|}{ Depression Score } \\
\hline & & & & & & PHQ2 & PHQ9 \\
\hline \multirow[t]{5}{*}{ Mpigi HCIV } & MP01 & 58 & Female & $\mid A$ & 10 & 5 & 14 \\
\hline & MP03 & 53 & Female & IA & 18 & 3 & 11 \\
\hline & MP10 & 39 & Female & IA & 8 & 4 & 12 \\
\hline & MP15 & 23 & Female & $\mid A$ & 7 & 3 & 19 \\
\hline & MP33 & 49 & Female & IA & 12 & 3 & 10 \\
\hline \multirow[t]{5}{*}{ Butoolo HCIII } & BT02 & 47 & Female & SA & 33 & 3 & 12 \\
\hline & BT07 & 45 & Female & SA & 33 & 6 & 16 \\
\hline & BT08 & 26 & Female & SA & 33 & 3 & 18 \\
\hline & BT12 & 32 & Female & SA & 33 & 4 & 15 \\
\hline & BT29 & 49 & Male & SA & 33 & 4 & 10 \\
\hline \multirow[t]{4}{*}{ Buwama HCIII } & BW08 & 53 & Female & IA & 8 & 5 & 20 \\
\hline & BW07 & 38 & Male & IA & 13 & 6 & 18 \\
\hline & BW03 & 61 & Male & IA & 14 & 6 & 15 \\
\hline & BW17 & 38 & Female & $\mid A$ & 14 & 6 & 14 \\
\hline \multirow[t]{4}{*}{ Nkozi Hospital } & NZ26 & 54 & Female & SA & 13 & 6 & 17 \\
\hline & NZ08 & 43 & Female & SA & 13 & 3 & 10 \\
\hline & NZ01 & 40 & Male & IA & 4 & 4 & 14 \\
\hline & NZ22 & 34 & Male & $\mid A$ & 13 & 4 & 15 \\
\hline
\end{tabular}

T PHQ9 and PHQ2 are screening instruments for depression, where: PHQ2 ranges from 0 to 6 and a value of zero indicates no depression, and a value greater than 3 indicates severe depression; PHQ9 ranges from 0 to 27 and a value of 0 -4indicates no depression, and a value greater than 14 indicates severe depression 
Table 2 Results of the pilot testing

\begin{tabular}{|c|c|c|c|c|c|}
\hline Item & Content & $\begin{array}{l}\text { Concern by } \\
\text { participants } \\
\text { (Y/N) }\end{array}$ & $\begin{array}{l}\text { Concern } \\
\text { by TCT } \\
\text { (Y/N) }\end{array}$ & Details of concern & Solution to concern \\
\hline Q1 & Daily activities & N & Y & Definition of health in Luganda & $\begin{array}{l}\text { Included term that acknowledges we mean mental } \\
\text { and physical health, not lifestyle }\end{array}$ \\
\hline Q2 & Social networks & N & N & & \\
\hline Q3 & Losing sleep & N & N & & \\
\hline Q4 & $\begin{array}{l}\text { Enjoying } \\
\text { recreation }\end{array}$ & N & Y & $\begin{array}{l}\text { Concept non-existent in traditional } \\
\text { local culture. Equivalent translation } \\
\text { hard to get }\end{array}$ & $\begin{array}{l}\text { Include an explanation that 'things that make you } \\
\text { happy' excludes anything with duty, like working or } \\
\text { caring activities }\end{array}$ \\
\hline Q5 & $\begin{array}{l}\text { Suitable } \\
\text { accommodation }\end{array}$ & $\mathrm{N}$ & $\mathrm{N}$ & & \\
\hline Q6 & $\begin{array}{l}\text { Neighbourhood } \\
\text { safety }\end{array}$ & N & N & & \\
\hline Q7 & $\begin{array}{l}\text { Potential for } \\
\text { assault }\end{array}$ & $\mathrm{N}$ & N & & \\
\hline Q8 & Discrimination & N & N & $\begin{array}{l}\text { Some respondents raised another } \\
\text { reason for discrimination not on the } \\
\text { provided list }\end{array}$ & $\begin{array}{l}\text { Added the option 'others' to the reasons for } \\
\text { discrimination }\end{array}$ \\
\hline Q9a & $\begin{array}{l}\text { Influencing local } \\
\text { decisions }\end{array}$ & N & $\mathrm{N}$ & & \\
\hline Q9b & $\begin{array}{l}\text { Freedom of } \\
\text { expression }\end{array}$ & N & $\mathrm{N}$ & & \\
\hline Q9C & $\begin{array}{l}\text { Appreciating } \\
\text { nature }\end{array}$ & Y & Y & $\begin{array}{l}\text { Concept non-existent in traditional } \\
\text { local culture. Equivalent translation } \\
\text { hard to get }\end{array}$ & Used locally appropriate examples \\
\hline Q9d & $\begin{array}{l}\text { Respect and } \\
\text { appreciation }\end{array}$ & N & N & & \\
\hline Q9e & $\begin{array}{l}\text { Love and } \\
\text { support }\end{array}$ & N & Y & $\begin{array}{l}\text { Luganda term for love has various } \\
\text { meanings }\end{array}$ & $\begin{array}{l}\text { Interviewer to be trained to use proper intonation of } \\
\text { the word }\end{array}$ \\
\hline Q9f & $\begin{array}{l}\text { Planning one's } \\
\text { life }\end{array}$ & N & N & & \\
\hline Q9g & $\begin{array}{l}\text { Imagination and } \\
\text { creativity }\end{array}$ & Y & Y & $\begin{array}{l}\text { Freedom of imagination and creativity } \\
\text { not traditionally expressed through } \\
\text { talent }\end{array}$ & $\begin{array}{l}\text { Opted for the expression my thoughts, feelings and } \\
\text { ideas, then added singing, dancing and talents as } \\
\text { examples }\end{array}$ \\
\hline Q9h & Access & Y & Y & $\begin{array}{l}\text { There was no equivalent of } \\
\text { 'Interesting' forms of activity/ } \\
\text { employment }\end{array}$ & Added locally appropriate examples \\
\hline
\end{tabular}

Particularly challenging was the translation of two English words which in Luganda have multiple meanings, with specific meaning being defined by the context: 'love' - 'okwagala', which can be loving, liking, and sex; and 'health' - 'embera y'obulamu' that can be 'lifestyle', 'nature of life' and 'standard of living'.

For example, the translation of Q9c (I am able to appreciate and value plants, animals and the world of nature) was difficult since this concept does not exist in the Kiganda culture. After discussion with the custodian of the OxCAP-MH, it was agreed that in order to maintain integrity and harmonised scoring of the OxCAP$\mathrm{MH}$, the Luganda translation of the question would be changed from the first forward translation - 'Nzisa ekitiibwa era manyi omugaso gw'ebiliime, ebisoolo n'ensi y'obutonde,' to 'Nnina obusoboozi obusiima ettaka lyaffe, amazzi (nga ogaseko amazzi g'enkuba) ebibiira, n'embela y'obudde,' which back translates to "I am able to appreciate our land, water (including rain water) forests and weather."

Q9g (I am free to use my imagination and to express myself creatively e.g. through art, literature, music, etc.) was also difficult since the concepts of imagination and creativity are not the same in the Ugandan context compared to a Western/European context, and neither is the option to indicate the existence of freedom of expression. The Translation Coordination Team suggested changing the wording to 'I am free to express what is in my heart' and give examples by adding opposing emotions 'such as when I am happy, when I am sad, when I an angry, when I am afraid, when I am excited.' After consultation with the custodian of the OxCAP-MH, the 
wording as 'I am free to express my thoughts, feelings and ideas (e.g. through singing, dancing, talking to someone, etc.)' was agreed, to maintain the originally intended content of 'imagination' and 'express myself creatively'.

Q9h (I have access to interesting forms of activity, or employment) was unclear for respondents as this concept does not exist in Kiganda culture. We suggested adding examples to the statement to make it culturally accessible. The final agreed text was 'I am able to participate in meaningful activities (or work), such as church activities, caring for family, hobbies, sports, etc.'

Two proposed changes involved phrases with potentially lost nuances, on the questions of neighbourhood safety and potential for assault. We contemplated if questions 6 (on safety) and 7 (on assault) were mutually exclusive. After consultations with both the experts and the custodian of the tool, we agreed that the two were mutually exclusive with Q6 referring to physical safety in neighbourhood and Q7 to different kinds of assault including safety at home, at work or in school, albeit being very broad in terms of type of violence and people who perpetrate (family, teacher, stranger). We therefore left both as they were.

One proposed change was an additional response option to Q8a on the expected reasons for discrimination. The respondents in the pilot study suggested adding 'economic status' as an option because it was the most common reason why they were discriminated against. Following discussions with the custodian of the tool, it was agreed that in order to keep the integrity of the original OxCAP-MH, the current answer options be kept, but an additional option of "Other", proceeded by space for respondents to write their answer, be adopted instead. This proposed change has also been included in the English, German and Hungarian versions (2020).

\section{Discussion}

The final official Luganda version of the OxCAP-MH was obtained following a systematic iterative process of cultural and linguistic validation and adaptation with the involvement of a multidisciplinary team. It is equivalent to the English version in content, but culturally acceptable, feasible and comprehensible by Luganda-speaking people. Although the tool was piloted among people with HIV and depression, it is suitable for use by all Luganda speakers. It is the first translation of the tool in a low-income country.

Our study followed a robust methodological design similar to Simon et al., 2018 [27], which reflects the principles of good practice for translation of PROMs adopted by ISPOR [27, 36]. Three extra steps were added to the recommended process: a validation workshop after the first back translations, which provided expert opinion on the anticipated acceptability and ease of use of the tool in the target population; a second forward translation of the edited phrases; and a confirmatory back translation of the second forward translation.

The differences in the cultural, social and political concepts between the UK where the tool was developed and Uganda were apparent in various questions. For example, in Q9a respondents are asked about their 'voice' in influencing local decisions. In Uganda the concept of local participation is not widespread. Other questions needed culturally appropriate examples: Q9c on appreciating nature and Q9g on using one's imagination. In Q8 a reason for discrimination was added. The cultural differences were pronounced in Q9b (freedom of expression), Q9c (appreciating nature), Q9g (imagination) and Q9h (access) as they proposed concepts that are culturally inexistent in Uganda. Capabilities questionnaires could be more flexible in the use of locally appropriate concepts. An alternative approach would be to develop a bottom-up list of locally relevant capabilities [39].

The tool was administered using two approaches. The interviewer-administered approach caters for both literate and illiterate respondents, despite being longer and more taxing in settings where many participants are involved. The self-administered approach is less repetitive and reduces interviewer fatigue. These advantages and disadvantages are specific for our context but might vary even across African settings. Prospective users should therefore choose and adapt a questionnaire delivery strategy that best fits their context.

Due to the cultural and contextual differences between the original European and the Ugandan setting, it was necessary to undertake an approach with multi-step consultations with the custodian of the tool, and with a team of anthropologists and social scientists. This study has highlighted the importance of following a systematic and standardised step-by-step linguistic and cultural adaptation process when transferring PROM instruments to different settings and languages. PROMs developed in a specific country/language cannot be taken "off-the-shelf" and used in a different setting.

Our study has a number of limitations. We were unable to employ an accredited professional company to translate the source English version to Luganda, however we engaged experienced and knowledgeable researchers. We believe this presents an opportunity, especially in LMICs, to translate and adopt the OxCAP-MH with limited resources, while still maintaining high standards of scientific rigor.

The OxCAP-MH is a capability-based measure that was developed around Nussbaum's ten central human capabilities. These represent aspects of quality of life that are considered the backbone for a life of human dignity that goes beyond survival and health and includes more complex components of what makes a life worth living, 
such as dignity, relationship, and discrimination. These core dimensions should be included in any exercise that assesses quality of life and human development across different populations. The Luganda version of the OxCAP-MH will provide an adequate framework for assessing the quality of life of people living with HIV/ AIDS and depression in Uganda. With this validation and adaptation exercise we have demonstrated that it is a transferable and suitable instrument for use in lowand middle-income country settings, with some of the aspects needing further explanations and amendments.

We recommend the use of the OxCAP-MH alongside generic HRQoL PROMs in economics evaluations until there is wider use of the OxCAP-MH within populations and in various settings, or in settings where comparison across disease conditions is sought. The OxCAP-MH will be used as a complementary PROM to the generic EQ-5D-5L in the economic evaluation of the HIV + D intervention for people with HIV and depression.

\section{Conclusion}

Our study developed a Luganda version of the OxCAP$\mathrm{MH}$ tool that is both culturally and linguistically appropriate for use in the Ugandan context and is feasible for the measurement of outcomes in people living with HIV with comorbid depression. The developed Luganda version of the OxCAP-MH measures broader wellbeing amongst Luganda speakers in Uganda using the capability approach, and can be used both as an alternative or as an addition to HRQoL measurement, both in mental health research and in research about people living with HIV with comorbid depression.

\section{Abbreviations \\ AIDS: Acquired Immuno-Deficiency Syndrome; BDI-II: Beck Depression Inventory 2nd edition; CRCT: Cluster Randomised Controlled Trial; DD: Depressive Disorders; DHO: District Health Officer; EQ 5D: EuroQol Five Dimension questionnaire; HIV: Human Immune Virus; HRQoL: Health-Related Quality of Life; IA: Interviewer-Administered; MoH: Ministry of Health; OxCAP- MH: Oxford CAPabilities Questionnaire for Mental Health; PLHIVs: People Living With HIV/AIDS; PROM: Patient-Reported Outcome Measure; PSI: Population Service International; SA: Self-Administered; SF-12: 12-item Short Form survey; SF-36: 36-item Short Form survey; SF-6: 6-item Short Form survey; TCT: Translation Coordination Team; TLV: Translation and Linguistic Validation; UK: United Kingdom}

\section{Acknowledgements}

We would like to thank the entire HIV + D trial team and the pilot study participants without whose support and cooperation this project would not have been possible. We would also want to thank the independent interpreters and the experts we consulted for their invaluable effort.

\section{Authors' contributions}

KRK, GG and YVL led the conceptualisation of this study; KRK wrote the first draft and led the write-up of the study; EK, YVL and AV wrote the main trial protocol and provided expert overall guidance on the study; KRK, YVL, GG and PT analysed the data; AL and JuS provided expert advice on the translation process and the instrument concept; PT coordinated the translation process and carried out pilot interviews; JoS carried out the initial translations and facilitated the validation workshop. All authors edited and commented on the manuscript. The author(s) read and approved the final manuscript.
Funding

The HIV + D trial is fully registered with trial registration number ISRC TN86760765, and the project was funded by a Senior Fellowship grant from the Wellcome Trust to Professor Eugene Kinyanda.

Availability of data and materials

Not Applicable.

\section{Declarations}

Ethics approvals and consent to participate

The pilot study was carried out after obtaining appropriate institutional and statutory ethical approvals. All study participants gave written informed consent, with those that couldn't sign using their fingerprint with a witness present.

Consent for publication

Not Applicable.

\section{Competing interests}

JuS has led the development of the OxCAP-MH measure.

\section{Author details}

${ }^{1}$ MRC/UVRI \& LSHTM Uganda Research Unit (Social Aspects of Health), Plot 51-59 Nakiwogo Road, Entebbe +256, Uganda. ${ }^{2}$ Centre for Health Economics in London, (Department of Global Health and Development), London School of Hygiene \& Tropical Medicine, London, UK. ${ }^{3}$ MRC/UVRI \& LSHTM Uganda Research Unit \& Senior Wellcome Trust Fellowship (Mental Health Section), Entebbe, Uganda. ${ }^{4}$ Butabika National Referral and Teaching Mental Hospital, Kampala, Uganda. ${ }^{5}$ Center for Public Health, (Department of Health Economics), Medical University of Vienna, Vienna, Austria. ${ }^{6}$ University of Oxford, (Department of Psychiatry and HERC), Oxford, UK.

Received: 18 November 2020 Accepted: 24 March 2021

Published online: 07 April 2021

\section{References}

1. Abas, M., Ali, G. C., Nakimuli-Mpungu, E., \& Chibanda, D. (2014). Depression in people living with HIV in sub-Saharan Africa: Time to act. Tropical Med Int Health, 19(12), 1392-1396. https://doi.org/10.1111/tmi.12382.

2. Kinyanda, E., Hoskins, S., Nakku, J., Nawaz, S., \& Patel, V. (2011). Prevalence and risk factors of major depressive disorder in HIV/AIDS as seen in semiurban Entebbe district, Uganda. BMC Psychiatry, 11(1), 205. https://doi.org/1 0.1186/1471-244X-11-205.

3. Kinyanda, E., Nakasujja, N., Levin, J., Birabwa, H., Mpango, R., Grosskurth, H., . Patel, V. (2017). Major depressive disorder and suicidality in early HIV infection and its association with risk factors and negative outcomes as seen in semi-urban and rural Uganda. J Affect Disord, 212, 117-127. https:// doi.org/10.1016/j.jad.2017.01.033.

4. Myer, L., Smit, J., Roux, L. L., Parker, S., Stein, D. J., \& Seedat, S. (2008), Common mental disorders among HIV-infected individuals in South Africa: Prevalence, predictors, and validation of brief psychiatric rating scales. AIDS Patient Care STDs, 22(2), 147-158. https://doi.org/10.1089/apc.2007.0102.

5. UNAIDS (2019). Global HIV and AIDS statistics.

6. Commission, U. A. (2015). The Uganda National HIV and AIDS Strategic Plan 2015/2016-2019/2020

7. Ministry of Health, U. (2016). Consolidated guidelines for the prevention and treatment of HIV in Uganda. (December 2016).

8. Douaihy, A., \& Singh, N. (2001). Factors affecting quality of life in patients with HIV infection. AIDS Read, 11(9), 450-454, 460-451, 475.

9. Farinpour, R., Miller, E. N., Satz, P., Selnes, O. A., Cohen, B. A., Becker, J. T., Visscher, B. R. (2003). Psychosocial risk factors of HIV morbidity and mortality: Findings from the multicenter AIDS cohort study (MACS). J Clin Exp Neuropsychol, 25(5), 654-670. https://doi.org/10.1076/jcen.25.5.654.14577.

10. Langford, S. E., Ananworanich, J., \& Cooper, D. A. (2007). Predictors of disease progression in HIV infection: a review. AIDS Res Ther, 4(1), 11. https:// doi.org/10.1186/1742-6405-4-11.

11. Kinyanda, E., Levin, J., Nakasujja, N., Birabwa, H., Nakku, J., Mpango, R., .. Patel, V. (2018). Major depressive disorder: Longitudinal analysis of impact on clinical and behavioral outcomes in Uganda. J Acquired Immune 
Deficiency Syndromes (1999), 78(2), 136-143. https://doi.org/10.1097/qai. 0000000000001647.

12. Springer, S. A., Dushaj, A., \& Azar, M. M. (2012). The impact of DSM-IV mental disorders on adherence to combination antiretroviral therapy among adult persons living with HIV/AIDS: a systematic review. AIDS Behav, 16(8), 21192143. https://doi.org/10.1007/s10461-012-0212-3.

13. Jenkinson, C., \& Morley, D. (2015). Patient reported outcomes. Eur J Cardiovasc Nurs, 15(2), 112-113. https://doi.org/10.1177/1474515115623407.

14. Seth, P., Patel, S. N., Sales, J. M., DiClemente, R. J., Wingood, G. M., \& Rose, E. S. (2011). The impact of depressive symptomatology on risky sexual behavior and sexual communication among African American female adolescents. Psychology, health \& medicine, 16(3), 346-356. https://doi.org/1 $0.1080 / 13548506.2011 .554562$.

15. Bhatia, M. S., \& Munjal, S. (2014). Prevalence of depression in people living with HIV/AIDS undergoing ART and Factors associated with it. J Clin Diagnostic Res, 8(10), WC01-WC04. https://doi.org/10.7860/JCDR/2014/772 5.4927.

16. Levintow, S. N., Pence, B. W., Ha, T. V., Minh, N. L., Sripaipan, T., Latkin, C. A., $\ldots$ Go, V. F. (2018). Prevalence and predictors of depressive symptoms among HIV-positive men who inject drugs in Vietnam. PLoS One, 13(1), e0191548. https://doi.org/10.1371/journal.pone.0191548.

17. Basch, E., Barbera, L., Kerrigan, C. L., \& Velikova, G. (2018). Implementation of patient-reported outcomes in routine medical care. Am Soc Clin Oncol Educ Book, 38(38), 122-134. https://doi.org/10.1200/edbk_200383.

18. Baumhauer, J. F. (2017). Patient-reported outcomes - Are they living up to their potential? N Engl J Med, 377(1), 6-9. https://doi.org/10.1056/NEJMp1 702978.

19. EuroQol (1990). A new facility for the measurement of health-related quality of life. Health Policy, 16(3), 199-208. https://doi.org/10.1016/0168-851 0(90)90421-9.

20. Ware Jr., J., Kosinski, M., \& Keller, S. D. (1996). A 12-item short-form health survey: Construction of scales and preliminary tests of reliability and validity. Med Care, 34(3), 220-233. https://doi.org/10.1097/00005650-19960300000003.

21. Ware Jr., J. E., \& Sherbourne, C. D. (1992). The MOS 36-item short-form health survey (SF-36). I Conceptual framework and item selection. Med Care, 30(6), 473-483.

22. Herdman, M., Gudex, C., Lloyd, A., Janssen, M., Kind, P., Parkin, D., ... Badia, X. (2011). Development and preliminary testing of the new five-level version of EQ-5D (EQ-5D-5L). Qual Life Res, 20(10), 1727-1736. https://doi.org/10.1 007/s11136-011-9903-x

23. Lenox-Smith, A., Macdonald, M. T. B., Reed, C., Tylee, A., Peveler, R., Quail, D., \& Wildgust, H. J. (2013). Quality of life in depressed patients in UK primary care: The FINDER study. Neurology and Ther, 2(1-2), 25-42. https://doi.org/1 0.1007/s40120-013-0006-1.

24. Shumye, S., Belayneh, Z., \& Mengistu, N. (2019). Health related quality of life and its correlates among people with depression attending outpatient department in Ethiopia: a cross sectional study. Health Qual Life Outcomes, 17(1), 169. https://doi.org/10.1186/s12955-019-1233-7.

25. Łaszewska, A., Schwab, M., Leutner, E., Oberrauter, M., Spiel, G., \& Simon, J. (2019). Measuring broader wellbeing in mental health services: Validity of the German language OxCAP-MH capability instrument. Qual Life Res, 28(8), 2311-2323. https://doi.org/10.1007/s11136-019-02187-9.

26. Lima, A. F. B. d. S., \& Fleck, M. P. d. A. (2011). Quality of life, diagnosis, and treatment of patients with major depression: a prospective cohort study in primary care. Brazilian Journal of Psychiatry, 33, 245-251, 3, DOl: https://doi. org/10.1590/S1516-44462011000300007.

27. Simon, J., Łaszewska, A., Leutner, E., Spiel, G., Churchman, D., \& Mayer, S. (2018). Cultural and linguistic transferability of the multi-dimensional OxCAP-MH capability instrument for outcome measurement in mental health: The German language version. BMC Psychiatry, 18(1), 173. https://doi. org/10.1186/s12888-018-1762-3.

28. Simon, J., Mayer, S., Łaszewska, A., Rugkåsa, J., Yeeles, K., Burns, T., \& Gray, A. (2020). Cost and quality-of-life impacts of community treatment orders (CTOS) for patients with psychosis: Economic evaluation of the OCTET trial. Soc Psychiatry Psychiatr Epidemiol, 56(1), 85-95. https://doi.org/10.1007/s0012 7-020-01919-4.

29. Lorgelly, P. K., Lorimer, K., Fenwick, E. A., Briggs, A. H., \& Anand, P. (2015). Operationalising the capability approach as an outcome measure in public health: The development of the OCAP-18. Soc Sci Med, 142, 68-81. https:// doi.org/10.1016/j.socscimed.2015.08.002
30. Nussbaum, A. S. a. M. (1993). The quality of life, (p. 30). Oxford: Clarendon. https://doi.org/10.1093/0198287976.001.0001.

31. Connell, J., O'Cathain, A., \& Brazier, J. (2014). Measuring quality of life in mental health: Are we asking the right questions? Social Science \& Medicine (1982), 120, 12-20. https://doi.org/10.1016/j.socscimed.2014.08.026.

32. Eugene Kinyanda, L. K., Richard S. Mpango, Christine Tusiime, Joshua Ssebunnya, Kenneth Katumba, Patrick Tenywa, James Mugisha, Geoffrey Taasi, Hafsa Sentongo, Dickens Akena, Yoko Laurence, Giulia Greco, Arpita Anand, Wilson Muhwezi, Helen A Weiss, Melissa Neuman, Anna Vassall, Birthe Knizek, Jonathan Levin, Pontiano Kaleebu, Vikram Patel (Forthcoming). Effectiveness and cost-effectiveness of integrating the management of depression into routine HIV Care in Uganda (the HIV+D trial): A cluster-randomised trial.

33. Baumgardt, J., Daum, M., von dem Knesebeck, O., Speck, A., \& Röh, D. (2018). Assess capabilities among chronically mentally ill people: First test results on a draft German version of the OxCAP-MH as part of the BAESCAP study. Psychiatr Prax, 45(3), 140-147. https://doi.org/10.1055/s-0043-101903.

34. Vergunst, F., Jenkinson, C., Burns, T., Anand, P., Gray, A., Rugkåsa, J., \& Simon, J. (2017). Psychometric validation of a multi-dimensional capability instrument for outcome measurement in mental health research (OxCAPMH). Health Qual Life Outcomes, 15(1), 250. https://doi.org/10.1186/s12955-01 7-0825-3.

35. Steel, C., van der Gaag, M., Korrelboom, K., Simon, J., Phiri, P., Baksh, M. F., ... Kingdon, D. (2015). A randomised controlled trial of positive memory training for the treatment of depression within schizophrenia. BMC Psychiatry, 15(1), 85. https://doi.org/10.1186/s12888-015-0453-6.

36. Wild, D., Grove, A., Martin, M., Eremenco, S., McElroy, S., Verjee-Lorenz, A., \& Erikson, P. (2005). Principles of good practice for the translation and cultural adaptation process for patient-reported outcomes (PRO) measures: Report of the ISPOR task force for translation and cultural adaptation. Value Health, 8(2), 94-104. https://doi.org/10.1111/j.1524-4733.2005.04054.x.

37. Vienna, D. O. H E a t M U o OxCAP-MH

38. Simon, J., Anand, P., Gray, A., Rugkåsa, J., Yeeles, K., \& Burns, T. (2013). Operationalising the capability approach for outcome measurement in mental health research. Soc Sci Med, 98, 187-196. https://doi.org/10.1016/j. socscimed.2013.09.019.

39. Greco, G., Skordis, J., Mkandawire, B. \& Mills, A. (2015). What is a good life? Selecting capabilities to assess women's quality of life in rural Malawi. Social Science \&amp Medicine, 130(C), 69-78

\section{Publisher's Note}

Springer Nature remains neutral with regard to jurisdictional claims in published maps and institutional affiliations.

\section{Submit your manuscript to a SpringerOpen ${ }^{\circ}$ journal and benefit from:}

- Convenient online submission

- Rigorous peer review

- Open access: articles freely available online

- High visibility within the field

- Retaining the copyright to your article

Submit your next manuscript at $\boldsymbol{\nabla}$ springeropen.com 